\title{
Assessment of adult rat cardiac fibroblast viability following chronic sunitinib treatment
}

\author{
McMullen, C. J., Wood, R. A., Cunningham, M. R., Currie, S \\ University of Strathclyde, United Kingdom
}

\begin{abstract}
Introduction The tyrosine kinase inhibitor sunitinib has dramatically improved cancer therapy in recent years however, this success has been marred by reports of associated cardiotoxicity. The effect of sunitinib on cardiac myocyte function has been extensively studied, yet little is known of wider ranging effects on cardiac non-myocytes. Cardiac fibroblasts (CF) are the most abundant cell type within the heart and are responsible for maintaining cardiac structure via extracellular matrix remodelling and for facilitating synchronised cardiac contraction. Here, we have investigated whether chronic sunitinib treatment adversely affects CF viability.
\end{abstract}

Methods CFs were isolated from adult male Sprague-Dawley rats (aged 10 weeks, weighing 350$450 \mathrm{~g}$ ) via bulk collagenase digestion and were maintained in culture before treatment with sunitinib (0.1-10 $\mu \mathrm{M}$ ) for 18 hour in the presence of serum (DMEM supplemented with $20 \% \mathrm{FBS}$ ). Cell phenotype and growth were imaged using a Leica EC3 digital camera affixed to a Leica DM IL LED inverted microscope. MTT assays and FACS were used to assess cell viability. Data is presented as mean values $\pm S D$ of $n$ observations, where $n$ represents the number of samples. Comparisons were assessed by one-way ANOVA with post hoc Dunnett's test.

Results Cell imaging indicated visible alterations in fibroblast phenotype following $3 \mu \mathrm{M}$ sunitinib treatment with significant cell loss at $10 \mu \mathrm{M}$. Assessment of CF viability via MTT assays showed a significant reduction in viable cells following treatment with $10 \mu \mathrm{M}$ sunitinib $(74.02 \% \pm 4.53 \%$ vs $94.12 \% \pm 6.95 \%$ (\% viable cells, sunitinib vs vehicle), $n=4, p=0.0002$ ). Further analysis via FACS confirmed these MTT findings, showing a dose-dependent reduction in the percentage of healthy cells following drug treatment $(44.00 \% \pm 14.49 \%$ vs $95.83 \% \pm 2.06 \%$ (\% healthy cells, $3 \mu \mathrm{M}$ sunitinib vs vehicle), $n=3, p=0.0001)$ and $(1.25 \% \pm 0.50 \%$ vs $95.83 \% \pm 2.06 \%(10 \mu \mathrm{M}$ sunitinib vs vehicle), $n=3$, $\mathrm{p}=0.0001)$. An increase in the number of late apoptotic $(17.56 \% \pm 13.38 \%$ vs $1.13 \% \pm 0.43 \%$ (\% late apoptotic cells, sunitinib vs vehicle), $\mathrm{n}=3, \mathrm{p}=0.004)$ and necrotic cells $(56.33 \% \pm 36.57 \%$ vs $1.69 \% \pm 2.55 \%$ (\% necrotic cells, sunitinib vs vehicle), $n=3, p=0.0002$ ) at $10 \mu \mathrm{M}$ sunitinib was also observed.

Conclusions Sunitinib causes a dose dependent decrease in CF viability with significant effects observed at concentrations as low as $3 \mu \mathrm{M}$. These results suggest that the cardiotoxic effects of sunitinib are likely to impact on both contractile and non-contractile cells of the heart. Further work is under way to assess how clinical doses can alter CF function. This may enhance understanding towards mechanisms related to sunitinib-induced cardiotoxicity.

Keywords: tyrosine kinase inhibitor; sunitinib; cardiac fibroblast

Accepted manuscript of the following research output: McMullen, C. J., Wood, R. A., Cunningham, M. R., \& Currie, S. (2019). Assessment of adult rat cardiac fibroblast viability following chronic sunitinib treatment. Heart , 105(suppl 4), A6-A7. https://doi.org/10.1136/heartinl-2019-SCF.13 